\title{
Hemosiderin in Intervillous Thrombus
}

National Cancer Institute

\section{Source}

National Cancer Institute. Hemosiderin in Intervillous Thrombus. NCI Thesaurus. Code C118151.

The deposition of hemosiderin in a focus of thrombus within the placental intervillous space. 